\title{
Experimental overview of DVCS and Generalized Parton Distributions at Jefferson Lab
}

\author{
Silvia Niccolai ${ }^{* \dagger}$ \\ IPN Orsay \\ E-mail: silvia@jlab.org
}

This paper focuses on recent promising results, obtained at Jefferson Lab, on cross sections and asymmetries for deeply virtual Compton scattering (DVCS), and their link to the Generalized Parton Distributions (GPDs). These data have opened the way to a "tomographic" representation of the structure of the nucleon, allowing the extraction of transverse-space distributions of the quarks at fixed longitudinal momentum. The extensive experimental program to measure GPDs at Jefferson Lab with the 12-GeV-upgraded electron accelerator is also outlined.

XXIV International Workshop on Deep-Inelastic Scattering and Related Subjects

11-15 April, 2016

DESY Hamburg, Germany

\footnotetext{
* Speaker.

${ }^{\dagger}$ For the CLAS Collaboration
} 


\section{Introduction: GPDs and DVCS}

Our understanding of nucleon structure is improving thanks to the ongoing intense effort of research on Generalized Parton Distributions (GPDs). These structure functions describe the correlations between the longitudinal momentum and transverse spatial position of the partons inside the nucleon, and, among other features, they give access to the contribution of the orbital angular momentum of the quarks and gluons to the spin of the nucleon $[1,2,3,4,5,6]$. The nucleon GPDs are accessible via measurements of various kinds of hard exclusive processes. Among these, the leptoproduction of a real photon, $\ell N \rightarrow \ell^{\prime} N^{\prime} \gamma$ (where the $\gamma^{*} N \rightarrow N^{\prime} \gamma$ subprocess is called deeply virtual Compton scattering, DVCS), is the reaction more directly interpretable in terms of GPDs.

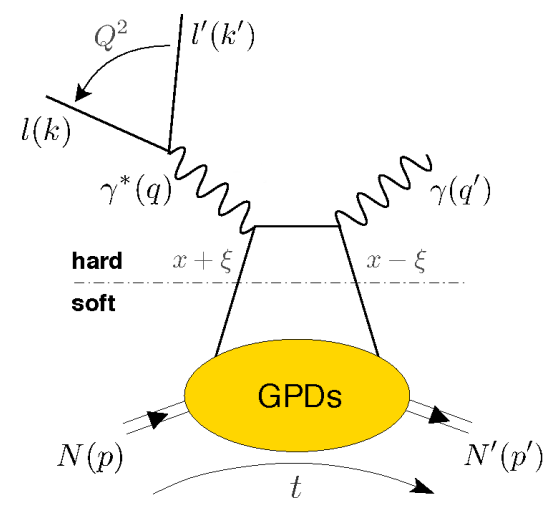

Figure 1: The "handbag" diagram for the DVCS process on the nucleon $\ell N \rightarrow \ell^{\prime} N^{\prime} \gamma$. The four-momenta of the incoming and outgoing leptons are labeled as $k$ and $k^{\prime}$, respectively, while those of the virtual and real photons are indicated by $q$ and $q^{\prime}$, respectively. $x$ is the average longitudinal momentum fraction of the struck parton. $t=\left(p-p^{\prime}\right)^{2}$ is the squared four-momentum transfer between the initial and final nucleon. $2 \xi$ is the difference between the longitudinal momentum fractions carried by the initial and the final parton. $\xi$ is proportional to the Bjorken variable $x_{B}\left(\xi \simeq \frac{x_{B}}{2-x_{B}}\right.$, where $x_{B}=\frac{Q^{2}}{2 M v}, M$ is the proton mass and $\left.v=E_{l}-E_{l^{\prime}}\right)$. $x$ is not accessible experimentally in the DVCS process.

Considering only the quark sector and quark-helicity conserving processes, which are dominant for DVCS, at leading order and at leading twist the reaction is described by four GPDs, $H^{q}$, $\tilde{H}^{q}, E^{q}, \tilde{E^{q}}$, (where $q$ denotes a light quark), which account for all the possible combinations of relative orientations of nucleon and parton helicity in the initial and final states. The GPDs $H$ and $E$ do not depend on the parton helicity and are therefore called unpolarized GPDs, while $\tilde{H}$ and $\tilde{E}$ depend on the parton helicity and are called polarized GPDs. The GPDs $H$ and $\tilde{H}$ conserve the helicity of the nucleon, whereas $E$ and $\tilde{E}$ correspond to nucleon-helicity flip transitions. At leading order in $\alpha_{s}$ and at leading twist, the GPDs depend upon three variables, $x$, $\xi$, and $t$.

DVCS observables can be expressed as linear and bilinear combinations of Compton Form Factors (CFFs), electromagnetic form factors, and terms depending on the kinematics. The CFFs are integrals of GPDs over the variable $x$. The study, at fixed $Q^{2}, x_{B},-t$, of the $\phi$ dependence of DVCS cross sections, cross-section differences, and beam or target spin asymmetries can help to extract the contributions from the various CFFs.

In the following, recent DVCS results produced at Jefferson Lab, and their impact on our knowledge of GPDs, will be discussed. 


\section{Hall-A cross sections}

Four-fold $\left(Q^{2}, x_{B},-t, \phi\right)$ differential cross sections, unpolarized and beam-polarized, for the exclusive electroproduction of photons were recently extracted at Jefferson Lab, by the Hall Collaboration, from a re-analysis of 2001 data (experiment E00-110 [11]). Polarized electrons, accelerated to $5.7572 \mathrm{GeV}$, impinged on a 15-cm-long liquid-hydrogen target. $e^{\prime} p^{\prime} \gamma$ events were collected for five different $\left(Q^{2}, x_{B}\right)$ kinematics, with fixed $Q^{2}$ but varying $x_{B}$. Each of these five $\left(Q^{2}, x_{B}\right)$ kinematics was further differentiated in $5-t$ bins and $24 \phi$ bins, where $\phi$ is the angle between the leptonic and the hadronic places, and four-fold unpolarized and beam-polarized cross sections were extracted.

The top part of Fig. 2 shows the obtained results for $Q^{2}=2.3 \mathrm{GeV}^{2}, x_{B}=0.36$, and $-t=0.32$ $\mathrm{GeV}^{2}$. The unpolarized cross sections peak towards $\phi=0^{\circ}$ due to the Bethe-Heitler (BH) process (dot-dashed gray curves), which has the same final state as DVCS, but the final photon is emitted by either the incoming or outgoing electron. The different contributions to the cross section (DVCS, $\mathrm{BH}$, Interference), shown in color, were separated, up to twist three for the interference term, using the BMK formalism [9]. This method also allowed the extraction of five observables (shown in the bottom panels of Fig. 2), which are linked to combinations of real and imaginary parts of CFFs.

The constant $Q^{2}$ dependence of the five observables (Fig. 2, bottom) confirms that the factorization and leading-twist approximations for DVCS are valid already at these relatively low $Q^{2}$ $\left(\sim 1-2 \mathrm{GeV}^{2}\right)$, as first observed in the previous analysis of these same data [11].

\section{CLAS cross sections}

The E01-113 experiment took place at JLab during three months in 2005, using the CEBAF 5.75-GeV polarized electron beam, a 2.5-cm-long liquid hydrogen target, and the Hall $\mathrm{B}$ large-acceptance CLAS spectrometer [13]. $e^{\prime} p^{\prime} \gamma$ events were detected in CLAS and in the Inner Calorimeter (IC), a specially designed electromagnetic calorimeter for low-angle photons. Tight cuts on missing masses and angles ensured the exclusivity of the final state. The data were divided into $21\left(Q^{2}, x_{B}\right)$ bins and $6-t$ bins. The events in each $\left(Q^{2}, x_{B},-t\right)$ bin were further divided into 24 bins in $\phi$.

The top panel of Fig. 3 shows, for one of the $21\left(Q^{2}, x_{B}\right)$ bins, the $\phi$-dependence of the $e p \rightarrow e^{\prime} p^{\prime} \gamma$ unpolarized cross section (top) and beam-polarized cross-section difference (bottom). For this $\left(Q^{2}, x_{B}\right)$ bin, three selected $-t$ bins are shown. As was observed in the Hall-A results (Fig. 2, top), the BH process (green long-dashed curves in Fig. 3) causes the unpolarized cross sections to peak towards $\phi=0^{\circ}$. The difference between the data and the BH curves can thus be attributed to the DVCS process. Figure 3 shows the calculations of four GPD models, described in the caption. Three of these models, VGG, KMS12, and the KM10a version of the KM model, describe the data well without additional inputs, while the KM10 model, which includes a sozeable contribution from the $\tilde{H}$ GPD, fails to reproduce the data near $\phi=180^{\circ}$. Therefore, the CLAS results support the expectation that $H$ dominates in the unpolarized ep $\gamma$ cross section.

The CLAS data were also used to extract the CFFs, using the procedure described in $[14,16]$, based on a local-fitting method at each given experimental $\left(Q^{2}, x_{B},-t\right)$ kinematic point. The bottom panel of Fig. 3 shows, for a selection of three out of the $21\left(Q^{2}, x_{B}\right)$ bins, the $t$ distribution of 

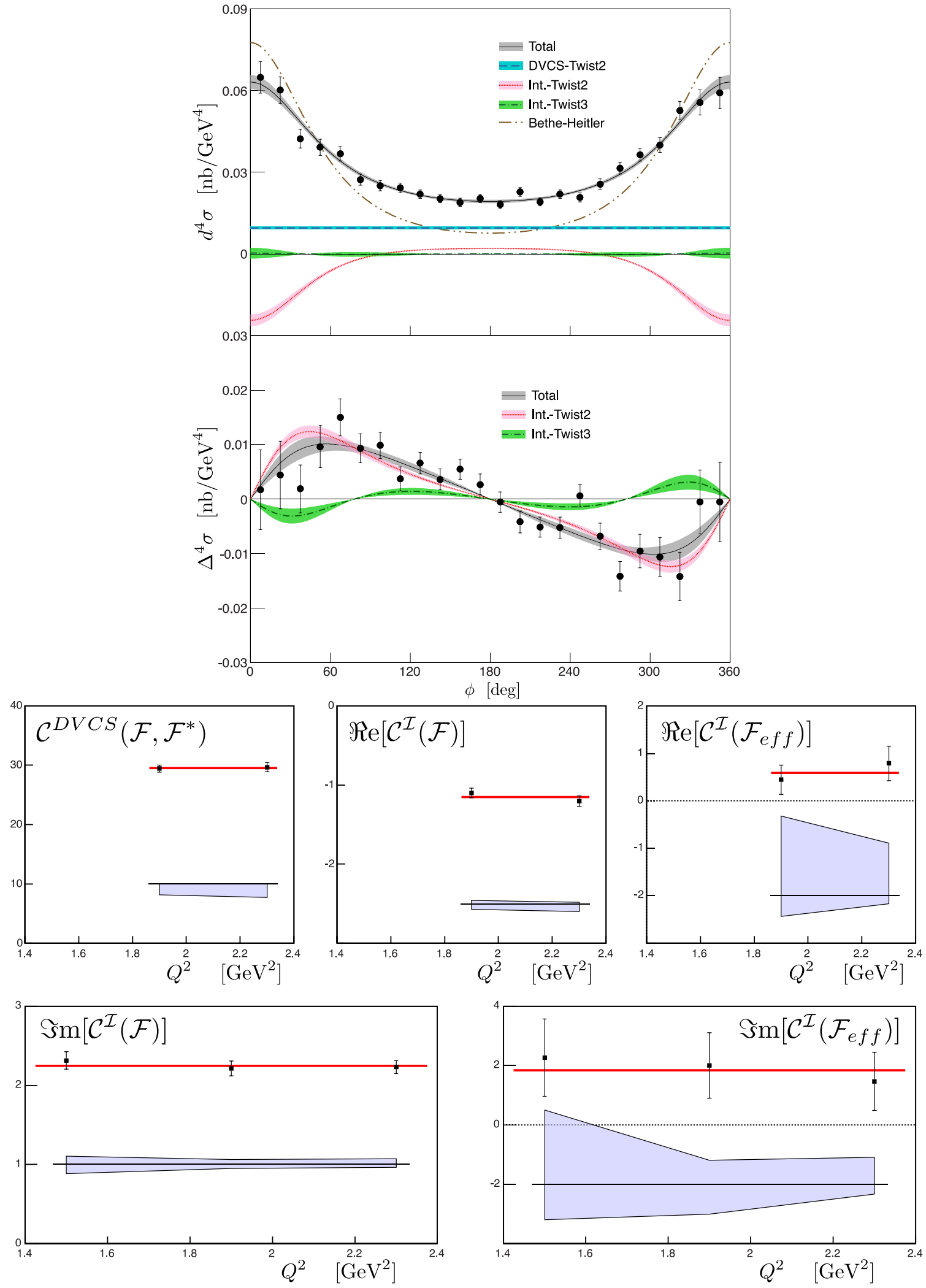

Figure 2: Top: Hall A results, for $Q^{2}=2.3 \mathrm{GeV}^{2}, x_{B}=0.36,-t=0.32 \mathrm{GeV}^{2}$ : unpolarized (top) and helicity-dependent (bottom) cross-section as functions of $\phi$. The error bars on the data points are statistical only. The shaded areas represent the statistical uncertainty for each contribution. Bottom: Combinations of effective CFFs extracted from the Hall-A data using the formalism developed in [9], integrated over $t$ and plotted as functions of $Q^{2}$. The top three plots show the effective CFFs resulting from the unpolarized cross section fit (for two kinematic bins), whereas the bottom plots show the effective CFFs resulting from the helicity-dependent cross section fit (for three kinematic bins). The shaded areas represent systematic uncertainties. 

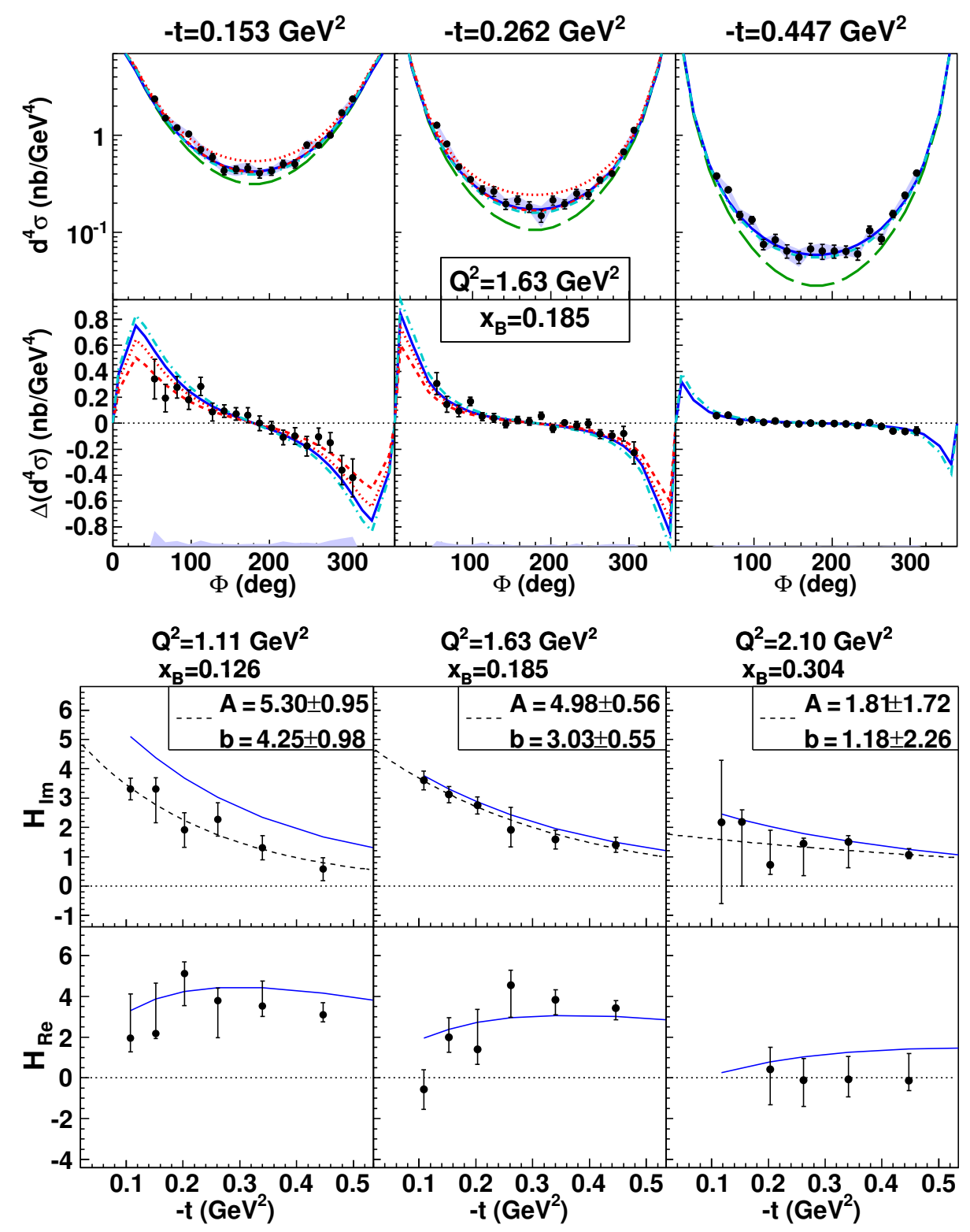

Figure 3: Top: CLAS results for the unpolarized cross section and beam-polarized cross-section difference for the $e p \rightarrow e^{\prime} p^{\prime} \gamma$ reaction, as a function of $\phi$, for $\left(Q^{2}, x_{B}\right)=\left(1.63 \mathrm{GeV}^{2}, 0.185\right)$ and for 3 values of $-t$ : $0.153,0.262$, and $0.447 \mathrm{GeV}^{2}$. The blue bands show the systematic uncertainties. The green long-dashed curves show the BH contribution only. The other curves correspond to the predictions of four GPD models: VGG [7, 8, 12, 17] (blue solid curves), KMS12 [18] (cyan dash-dotted curves), and two versions of the KM (Kumericki-Müller) model [19, 21], KM10 (red dotted curves) and KM10a (red short-dashed curves). Bottom: Results of the CFF fit of the CLAS unpolarized and beam-polarized cross sections for $H_{I m}$ (upper panel) and $H_{R e}$ (lower panel), for three $\left(Q^{2}, x_{B}\right)$ bins, as a function of $t$. The blue solid curves are the predictions of the VGG model. The black dashed curves show the fit of the results by the function $A e^{b t}$. 
the fitted $H_{I m}$ and $H_{R e}$. Figure 3 (bottom) also shows the predictions of the VGG model, which overestimates the fitted $H_{I m}$ at the smallest values of $x_{B}$. The $t$-dependence of $H_{I m}$ was fitted with the function $A e^{b t}$, with $A$ and $b$ left as free parameters. Keeping in mind that $b$ is related to the transverse size of the nucleon, the fact that $b$ increases when $x_{B}$ descreases suggests that the nucleon size increases at lower parton-momentum values, thus revealing from experiment a first tomographic image of the nucleon. The increase of $A$, instead, suggests that the density of partons in the proton increases as $x_{B}$ increases.

\section{Longitudinal target asymmetries from CLAS}

The CLAS Collaboration recently released results for four-fold differential single and double spin asymmetries for the $e p \rightarrow e^{\prime} p^{\prime} \gamma$ reaction, using a longitudinally polarized proton target. The data, part of the E05-114 experiment which ran in Hall B at Jefferson Lab during the year 2009 , were produced by a $5.9-\mathrm{GeV}$ polarized electron beam impinging onto a solid ammonia target $\left(\mathrm{NH}_{3}\right)$, polarized along the beam direction [23], and were collected by the CLAS detector and the IC. Exclusive $e^{\prime} p^{\prime} \gamma$ events were selected, and for each $\left(Q^{2}, x_{B},-t\right)$ bin, beam-, target-, and double spin asymmetries (denoted with BSA, TSA, and DSA, respectively) were extracted as functions of $\phi$, in ten $\phi$ bins.

Figure 4 shows the TSA at $\phi=90^{\circ}$, as a function of $-t$, for the five measured $\left(Q^{2}, x_{B}\right)$ bins, compared to four GPD models, described in the caption. The amplitude of the target-spin asymmetry seems rather constant as a function of all kinematic variables, $-t$ included, apart from the expected systematic drop at $t$ approaching $t_{\min }$. Aside from the highest- $x_{B}$ point, where VGG reproduces well the data, the rather flat $-t$ shape of the target-spin asymmetry is clearly not correctly predicted well by the VGG, GGL, and KMS12 models. These models approximately reproduce the low- $t$ magnitude of the asymmetry in some kinematics. Overall, the data agree best with the predictions of the KMM12 model (for the bins where this model is applicable). The sixth panel of Fig. 4 shows the comparison of the CLAS TSA, at $\phi=90^{\circ}$, integrated over $Q^{2}$ and $x_{B}$, with previous data from HERMES [26] and CLAS [27]: the E05-114 results improve the existing statistics by more than a factor of 5 in the $-t$ region up to $\sim 0.4(\mathrm{GeV} / \mathrm{c})^{2}$, and extend the $-t$ range up to 1.6 $(\mathrm{GeV} / \mathrm{c})^{2}$.

The three sets of asymmetries (BSA, TSA and DSA) for all kinematic bins were processed using the previously mentioned fitting procedure to extract the CFFs. Figure 5 shows $H_{I m}$ (black full squares) and $\tilde{H}_{I m}$ (red full circles), which are obtained from the fit of the CLAS E05-114 asymmetries, as a function of $-t$ for each of the $5\left(Q^{2}, x_{B}\right)$ bins. These are the two CFFs that appear to be better constrained by these data. It appears that the $t$-slope of $H_{I m}$ is much steeper than that of $\tilde{H}_{I m}$, hinting that the axial charge (linked to $\tilde{H}_{I m}[16]$ ) might be more "concentrated" in the center of the nucleon than the electric charge (linked to $H_{I m}[16]$ ).

\section{12-GeV upgrade of Jefferson Lab}

An energy upgrade to $12 \mathrm{GeV}$ of the CEBAF accelerator at JLab was completed in 2014, and the capabilities of the detectors in the Halls A, B, and C are being enhanced to suit the new experimental program, which will have a specific focus on the study of GPDs. Covering a range in 


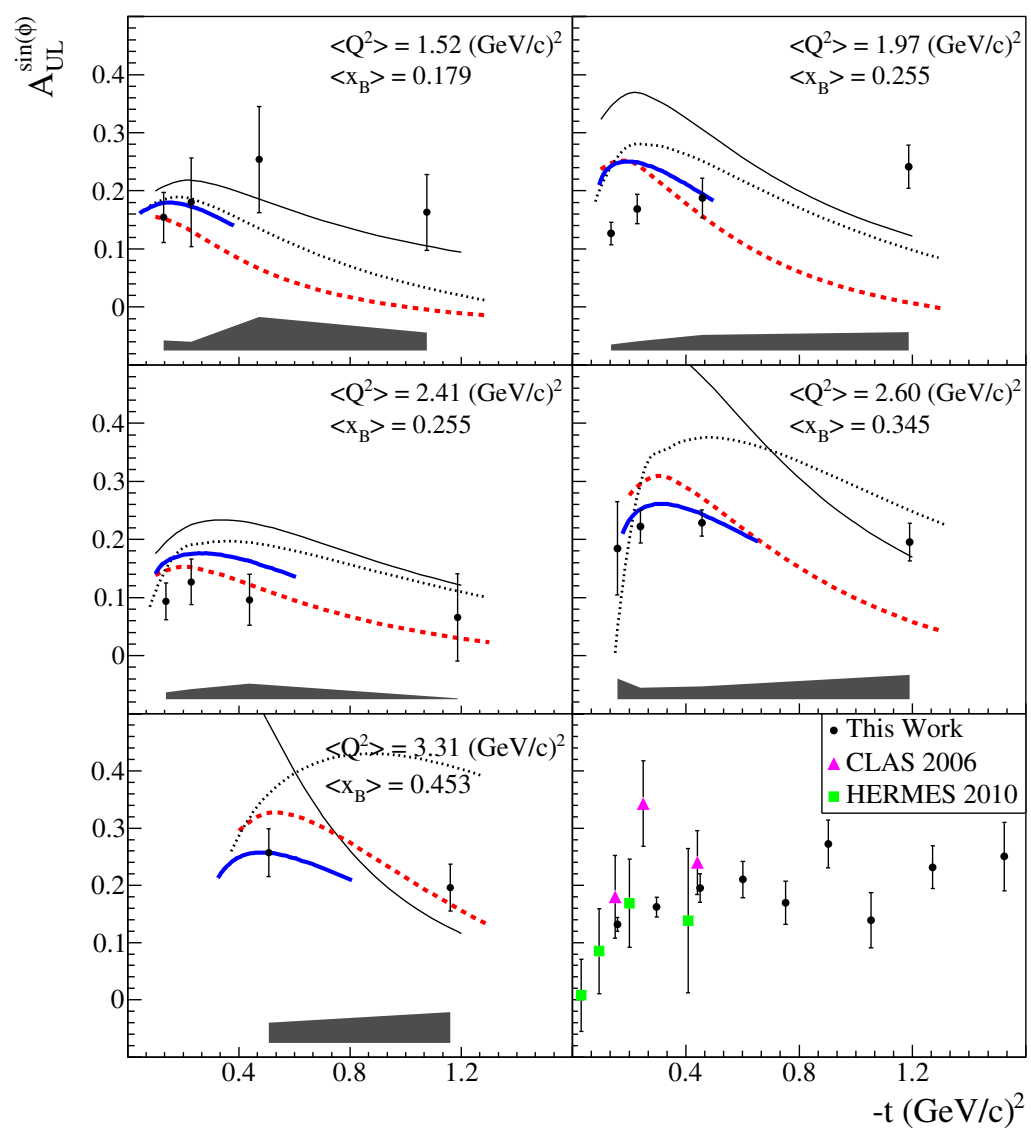

Figure 4: First five plots: $-t$ dependence of the the TSA at $\phi=90^{\circ}$ for five $\left(Q^{2}, x_{B}\right)$ bins. The shaded bands represent the systematic uncertainties. The curves show the predictions of four GPD models: i) VGG [7, 8] (red-dashed), ii) KMS12 [18] (black-dotted), KMM12 [20] (blue-thick solid), GGL [24, 25] (black-solid). Sixth plot: comparison of the TSA at $\phi=90^{\circ}$ as a function of $-t$ for the CLAS E05-114 results (black dots) integrated over all $Q^{2}$ and $x_{B}$ values $\left(\left\langle Q^{2}\right\rangle=2.4 \mathrm{GeV}^{2},\left\langle x_{B}\right\rangle=0.31\right.$ ) with the HERMES results [26] (green squares) at $\left\langle Q^{2}\right\rangle=2.459 \mathrm{GeV}^{2},\left\langle x_{B}\right\rangle=0.096$, and with the previously published CLAS results [27] (pink triangles), at $\left\langle Q^{2}\right\rangle=1.82 \mathrm{GeV}^{2},\left\langle x_{B}\right\rangle=0.28$.

$x_{B}$ from 0.1 to 0.7 and in $Q^{2}$ from 1 to $10 \mathrm{GeV}^{2}$, the upgraded JLab will be very well suited to study GPDs in the valence regime. The first experiment of the $12-\mathrm{GeV}$ era is presently running in Hall A and focuses on proton DVCS: beam-polarized and unpolarized cross sections will be measured with high precision at three electron-beam energies to increase the kinematical coverage of the previous measurements [11], perform an accurate $Q^{2}$-scaling test, and extract the squared-DVCS and the interference terms of the cross section via a Rosenbluth separation. The experimental program for the first 5 years of operation of CLAS12 (the new Hall-B detector) will be focused on measurements of GPDs in exclusive processes. Measurements of BSAs, unpolarized and polarized proton-DVCS cross sections, as well as TSA and DSA with longitudinally polarized targets and single and double spin asymmetries on transversely polarized targets are planned. A similar experimental program will be carried out on the neutron. The DVCS experiments planned at JLab at $12 \mathrm{GeV}$ have the 

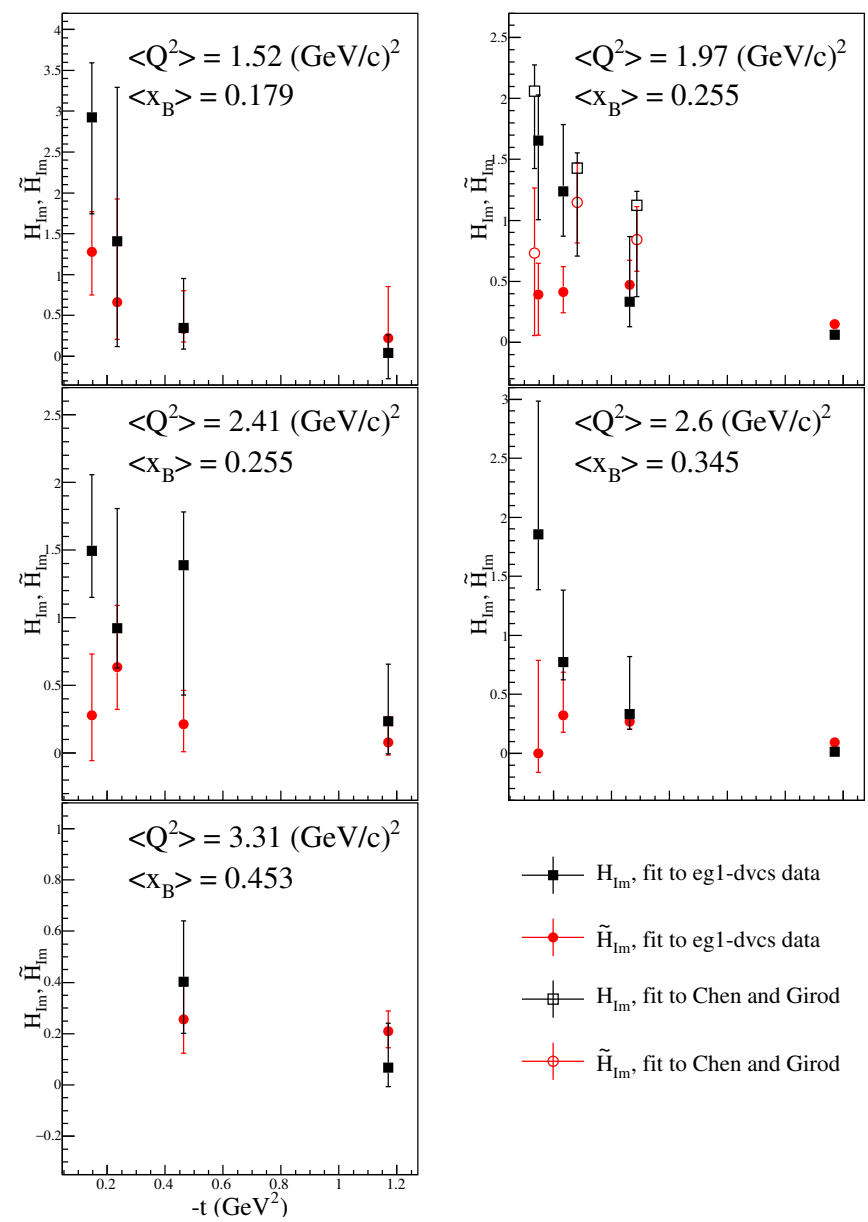

$$
\begin{aligned}
& \mathrm{H}_{\mathrm{Im}} \text {, fit to eg1-dves data } \\
& \varnothing-\tilde{\mathrm{H}}_{\mathrm{Im}} \text {, fit to eg1-dvcs data } \\
& \text {, fit to Chen and Girod }
\end{aligned}
$$

Figure 5: $t$ dependence for each $\left(Q^{2}, x_{B}\right)$ bin of $H_{I m}$ (black squares) and $\tilde{H}_{I m}$ (red circles). The solid points are obtained from fits to the E05-114 data (TSA, BSA and DSA) [29]. The open points were obtained from fits to the BSA results from [15] integrated over all values of $Q^{2}$ at $x_{B} \sim 0.25$, and the TSAs from [27].

ultimate goal to achieve a model-independent measurement of quark-flavor separated CFFs. The flavor separation of the CFFs will represent a major step forward towards the unraveling of the contribution of the quarks' angular momentum to the total nucleon spin via Ji's sum rule [3].

\section{References}

[1] D. Müller et al., Fortschr. Phys. 42, 101 (1994).

[2] A.V. Radyushkin, Phys. Rev. D 56, 5524 (1997).

[3] X.-D. Ji, Phys. Rev. Lett. 78, 610 (1997).

[4] M. Diehl, Eur. Phys. J. C 25, 223 (2002).

[5] A.V. Belitsky, D. Müller, and A. Kirchner, Nucl. Phys. B 629, 323 (2002).

[6] A.V. Belitsky and A.V. Radyushkin, Phys. Rept. 418, 1 (2005). 
[7] M. Vanderhaeghen, P.A.M. Guichon, and M. Guidal, Phys. Rev. Lett. 80, 5064 (1998).

[8] M. Vanderhaeghen, P.A.M. Guichon, and M. Guidal, Phys. Rev. D60, 094017 (1999).

[9] A.V. Belitsky and D. Müller, Phys. Rev. D82, 074010 (2010).

[10] M. Defurne et al. (Hall-A Collaboration), Phys. Rev. C92, 055202 (2015).

[11] C. Munoz-Camacho et al. (Hall-A Collaboration), Phys. Rev. Lett. 97, 26002 (2006).

[12] K. Goeke, M. Polyakov, and M. Vanderhaeghen, Prog. Part. Nucl. Phys. 47, 401 (2001).

[13] B.A. Mecking et al., Nucl. Instrum. Meth. A503, 513 (2003).

[14] M. Guidal, Eur. Phys. J. A37, 319 (2008).

[15] F.X. Girod et al. (CLAS Collaboration), Phys. Rev. Lett. 100, 162002 (2008).

[16] M. Guidal, H. Moutarde, and M. Vanderhaeghen, Rept. Prog. Phys. 76, 066202 (2013).

[17] M. Guidal, M.V. Polyakov, A.V. Radyushkin, and M. Vanderhaeghen, Phys. Rev. D72, 054013 (2005).

[18] P. Kroll, H. Moutarde, and F. Sabatie, Eur. Phys. J. C73, 1, 2278 (2013).

[19] K. Kumericki and D. Mueller, Nucl. Phys. B841, 1 (2010).

[20] K. Kumericki, D. Mueller, and M. Murray, Phys. Part. Nucl. 45, 4, 723 (2014).

[21] K. Kumericki et al., arXiv:1105.0899 [hep-ex].

[22] H.S. Jo et al. (CLAS Collaboration), Phys. Rev. Lett. 115, 21, 212003 (2015).

[23] C.D. Keith et al., Nucl. Instrum. Meth. A501, 327 (2003).

[24] G.R. Goldstein, J. O. Gonzalez-Hernandez, and S. Liuti, Phys. Rev. D84, 034007 (2011).

[25] J. O. Gonzalez-Hernandez, S. Liuti, G.R. Goldstein, and K. Kathuria, Phys. Rev. C88, 6, 065206 (2013).

[26] A. Airapetian et al. (HERMES Collaboration) JHEP 06, 019 (2010).

[27] S. Chen et al. (CLAS Collaboration), Phys. Rev. Lett. 97, 072002 (2006).

[28] E. Seder et al. (CLAS Collaboration), Phys. Rev. Lett. 114, 3, 032001 (2015); addendum: Phys. Rev. Lett. 114, 8, 089901(2015).

[29] S. Pisano et al. (CLAS Collaboration), Phys. Rev. D91, 5, 052014 (2015). 